\title{
INAPTIDÃO DE CANDIDATOS À DOAÇÃO DE SANGUE RELACIONADA À SOROPOSITIVIDADE PARA INFECÇÃO CHAGÁSICA NAS DIFERENTES REGIÕES DO BRASIL
}

INABILITY OF CANDIDATES FOR BLOOD DONATION RELATED WITH A

SEROPOSITIVITY FOR CHAGASIC INFECTION IN DIFFERENT REGIONS OF BRAZIL

\section{Milce Costa}

Doutora em Medicina Tropical. Docente da FACER Faculdades Unidade Ceres - GO, Brasil. E-mail-milcebiomol@yahoo.com.br

Endereço para correspondência: Av. Brasil, S/N, Qd. 13, Morada Verde, Ceres - Go, CEP: 76300-000. Fone/Fax: (62) 3323-1040

\section{Juliane dos Santos Silva}

Curso de Enfermagem - FACER Faculdades Unidade de Ceres-GO, Brasil.

E-mail-juliane-santos@outlook.com

\section{Valkíria dos Reis Carvalho Muniz}

Curso de Enfermagem - FACER Faculdades Unidade de Ceres-GO, Brasil.

E-mail - val-gustavo2010@ hotmail.com

RESUMO: Introdução: A doença de Chagas é uma infecção sistêmica de evolução crônica que tem como agente etiológico o protozoário monoflagelado Trypanosoma cruzi. A transmissão por transfusão sanguínea tem importante relevância epidemiológica, uma vez que corresponde de 5 a 20\% dos casos descritos na literatura. Objetivo: Analisar a prevalência de inaptidão em candidatos à doação de sangue com sorologia positiva para Chagas por regiões do Brasil entre os anos de 2000 a 2013. Metodologia: Trata-se de uma revisão bibliográfica, de aspecto quantitativo. Resultados e Discussão: As maiores prevalências de doadores inaptos com sorologia positiva para doença de Chagas ocorreram na região Nordeste, especificamente na cidade de Iguatu-CE e na região Sudeste, na cidade de Patos de MinasMG, com percentuais de $1,90 \%$ e $1,20 \%$ respectivamente. Diante destes dados pode-se presumir que nestas localidades não houve triagem sorológica adequada no serviço de hemoterapia bem como o combate ao vetor apresentou falhas. A região Sudeste foi a que apresentou a maioria das prevalências abaixo do índice de prevalência de doadores chagásicos, tal situação pode ser justificada pelo fato das cidades pesquisadas não serem regiões endêmicas para doença de Chagas, bem como pelo combate sistemático ao vetor nestes locais. Conclusão: A realização de pesquisas verificando doadores inaptos com sorologia positiva para doença de Chagas em bancos de sangue nas diferentes regiões do Brasil é de extrema relevância para avaliar a situação transfusional peculiar dos hemocentros de cada região com o intuito de traçar estratégias locais para eliminar a transmissão da doença de Chagas por transfusão sanguínea.

Palavras-chaves: Doença de Chagas. Transfusão sanguínea. Doadores de sangue. Prevalência. Trypanosoma cruzi. 
ABSTRACT: Introduction: Chagas disease is a systematic infection of chronic evolution that has as an etiologic agent the protozoan Trypanosoma cruzi. The transmission through blood transfusion has important epidemiological relevance once it corresponds from 5 to $20 \%$ of the cases described in the literature. Objective: To analyze the prevalence of inability in candidates to the blood donation with positive serology for Chagas for the Brazilian regions between the years of 2000 and 2013. Methodology: It deals with a bibliographic review, of quantitative aspect. Results and discussion: The biggest prevalence of inapt donators with positive serology for Chagas disease have occurred in the Northeast region, specifically in Iguatu town, Ceará State and in the Southeast region in Patos de Minas -MG town, Minas Gerais State, with percentage of $1.90 \%$ and $1.20 \%$ respectively. Before these data we can presume that in these locations have not had Serological screening adequate in the service of hemotherapy as well as the combat to the vector has shown failures. The Southeast region has been the one that has shown the majority of the prevalence below the index of prevalence of donators with Chagas, such situation can be justified by the fact of the cities researched not to be endemic regions for Chagas disease, as well as for the systematic combat to the vector in these locations. Conclusion: The conducting of researches verifying inapt donators with positive serology for Chagas disease in blood bank in the different regions of Brazil is of extreme relevance to evaluate the transfusion situation peculiar of the hemo-centers of each region with the intension of drawing local strategies to eliminate the transmission of Chagas disease through blood transfusion.

Keywords: Chagas disease. Blood transfusion. Blood donators. Prevalence. Trypanosoma cruzi 


\section{INTRODUÇÃO}

A doença de Chagas, também conhecida por tripanossomíase americana, é uma infecção sistêmica de evolução crônica que tem como agente etiológico o protozoário monoflagelado Trypanosoma cruzi. A transmissão da doença de Chagas pela via vetorial era considerada, até a década de 70, o principal mecanismo de transmissão, correspondendo a $80 \%$ dos casos da doença, no entanto, outras formas de transmissão como a via oral, vertical, acidental e transplantar devem ser consideradas. A transmissão por transfusão sanguínea tem importante relevância epidemiológica, uma vez que varia entre de 5 a $20 \%$ dos casos descritos na literatura a partir da década de 80 , quando foram implantadas medidas de controle ao vetor (ARAS et al., 2003; MELO et al., 2009; MORAES-SOUZA; FERREIRA-SILVA, 2011; SILVA; LUNA, 2013).

A descoberta de transmissão da doença de Chagas por meio de transfusão de sangue foi relatada no Brasil pela primeira vez em 1952 quando a sorologia pré-transfusional não era obrigatória. O processo de mudança da população do campo para a cidade devido à industrialização no Brasil, na década de 50, ocasionou a urbanização da doença, causando alta prevalência de doadores com a doença de Chagas nos hemocentros do país, alcançando índices de aproximadamente 10\%. Os altos índices de indivíduos chagásicos nas grandes cidades e a falta de programas de controle, contribuiu para que a transmissão transfusional causasse na década de 70, aproximadamente 20 mil novos casos da doença no Brasil (DIAS 2006; FITARELLI; HORN, 2009; MORAES-SOUZA; FERREIRA-SILVA, 2011).

Clinicamente a doença de Chagas apresenta duas fases bem distintas entre si: fase aguda e fase crônica. A fase crônica é subdividida em crônica assintomática (forma indeterminada ou latente) e crônica sintomática. A forma indeterminada ou latente da doença é caracterizada pela inexistência de manifestações clínicas e achados laboratoriais significativos. No entanto, nesta fase a sorologia é reagente e normalmente é diagnosticada em bancos de sangue. Sendo assim, essa é a forma da doença de Chagas mais comumente identificada na população das áreas endêmicas e entre doadores de sangue (MEYER; KANESHIMA; SOUZA-KANESHIMA， 2006; BARBOSA， 2009; SANTOS，2011; LOZANO, 2011).

Quando a doença de Chagas é transmitida por transfusão sanguínea para indivíduos imunocompetentes a doença apresenta-se oligo ou assintomaticamente na maioria dos casos. De forma semelhante à transmissão vetorial, a infecção chagásica adquirida pela via transfusional raramente se manifesta na fase aguda em imunocompetentes. O período de 
incubação nestes casos costuma ser mais longo, chegando a exceder 100 dias. Já em pacientes imunocomprometidos como os HIV positivos, transplantados e pacientes em tratamento com quimioterápicos a fase aguda da doença pode se manifestar de modo excepcional. As manifestações nestes pacientes são caracterizadas por febre prolongada, linfadenopatia e hepatoesplenomegalia (MORAES-SOUZA; FERREIRA-SILVA, 2011).

Priorizando a segurança da qualidade dos produtos sanguíneos que serão usados em transfusões, os hemocentros devem seguir um conjunto de medidas chamado de ciclo hemoterápico. Neste ciclo é realizada a triagem clínica, epidemiológica, sorológica e imunohematológica, em seguida o processamento, fracionamento das unidades coletadas, dispensação, transfusão e avaliação pós-transfusão (CARRAZONE; BRITO; GOMES, 2004).

Com o intuito de investigar a soropositividade de tripanossomíase nos bancos de sangue, as etapas do ciclo hemoterápico devem ser observadas rigorosamente. Normalmente na triagem sorológica e imunológica são aplicados os testes sorológicos usando antígenos $T$. cruzi: Hemaglutinação indireta (HAI), Imunofluorescência indireta (IFI) e Método imunoenzimático (ELISA) (LUNARDELLI, et al., 2007). Entretanto, a ANVISA na RDC 57/2010 define a necessidade de realizar apenas uma única técnica de alta sensibilidade para a detecção de anticorpo anti-T. cruzi na triagem sorológica dos doadores de sangue (ANVISA, 2010).

Além das medidas já preconizadas e adotadas para assegurar a qualidade da triagem clínica e sorológica dos candidatos à doação de sangue foram implantadas importantes políticas nacionais de controle da infecção chagásica no Brasil pelo Ministério da Saúde como o Programa Nacional de Sangue e Hemoderivados em 1980. Consequentemente foi implantada uma rede pública estadual hierarquizada de serviços de hemoterapia, a HEMORREDE, gerando recursos humanos qualificados, contribuindo para a redução da prevalência de sorologia positiva para T. cruzi entre os doadores do país de 6,9\% para 0,2\% em aproximadamente 30 anos (MORAES-SOUZA; FERREIRA-SILVA, 2011).

$\mathrm{Na}$ atualidade existem poucos trabalhos científicos publicados sobre a prevalência de candidatos à doação de sangue inaptos com sorologia positiva para doença de Chagas no Brasil. Determinar essa prevalência pode ser um indicador interessante para avaliar o risco da doença de Chagas transfusional bem como para mensurar o nível de transmissão da doença em uma determinada região (SAÚDE-GUIMARÃES; FARIA, 2007). Dessa forma, o presente estudo teve por objetivo analisar a prevalência de inaptidão em candidatos à doação de sangue com sorologia positiva para Chagas por regiões do Brasil em artigos publicados entre 2000 a 2013 com o intuito de verificar os índices descritos na literatura pesquisada. 


\section{METODOLOGIA}

Trata-se de uma revisão bibliográfica, de aspecto quantitativo relacionado à inaptidão de candidatos à doação de sangue com soropositividade para doença de Chagas.

Este trabalho foi realizado através de consulta em base de dados na biblioteca virtual de saúde (BVS), SciELO, LILACS, BIREME e PUBMED por meio de periódicos científicos, dissertações e teses referentes ao assunto abordado tendo como descritores: doença de Chagas, Transfusão sanguínea, Doadores de sangue, Prevalência, T. cruzi. Os textos foram analisados de forma completa, sendo selecionados os que correspondiam ao tema abordado.

Os critérios de inclusão utilizados correspondiam aos artigos publicados no idioma português e na língua inglesa que abordavam o tema proposto com publicações que datavam entre os anos de 2000 a 2013. Levando-se em conta esse critério, dos 13 artigos selecionados para verificar a prevalência da inaptidão de candidatos à doação de sangue com soropositividade para doença de Chagas nas diferentes regiões do Brasil, foi possível analisar um intervalo de tempo compreendido entre anos de 1995 a 2010. Neste estudo foram excluídos os artigos publicados que datam de período inferior ao designado, 2000 a 2013, e que não abordavam a temática proposta.

Para composição da tabela relacionada à prevalência de doadores inaptos com sorologia positiva para doença de Chagas por regiões do Brasil, foram utilizados 13 artigos científicos que nos possibilitaram selecionar estados correspondentes a três regiões do Brasil que abordavam o tema proposto (Nordeste, Sudeste e Sul). Nesta tabela está disponibilizada a quantidade de doações realizadas bem como a prevalência de doadores inaptos com sorologia positiva para doença de Chagas.

\section{RESULTADOS E DISCUSSÃO}

A doença de Chagas representa uma das mais importantes ocorrências de saúde pública. De acordo com a Word Health Organization (WHO) existem aproximadamente 10 milhões de pessoas infectadas com o T. cruzi no mundo, especialmente na América Latina. Aproximadamente 25 milhões de pessoas vivem em áreas de risco para transmissão da doença ocasionando mais de 10.000 mortes, segundo levantamento realizado no ano de 2008 (WHO, 2010). Dessa forma, inúmeros esforços vêm sendo realizados visando à eliminação da transmissão domiciliar pelo Triatoma infestans desde a década de 70. Tal empenho resultou 
ao Ministério da saúde, no ano de 2006, uma certificação internacional concedida pela Organização Pan Americana de Saúde (OPAS) onde o Brasil foi considerado livre momentaneamente da transmissão da doença especificamente pelo triatomíneo da espécie $T$. infestans (RAMOS; CARVALHO, 2001; BRASIL, 2006).

Em relação aos serviços de hemoterapia, o Ministério da Saúde também criou programas para melhorar a qualidade da triagem clínica e sorologia dos candidatos a doação de sangue. Inicialmente foi idealizado o Programa Nacional de Sangue e Hemoderivados e posteriormente em 1980, houve a implantação da HEMORREDE, uma rede pública estadual hierarquizada de serviços de hemoterapia, atraindo assim recursos humanos qualificados, contribuindo para a redução da prevalência de positividade para $T$. cruzi entre os doadores (MORAES-SOUZA; FERREIRA-SILVA, 2011).

Mesmo após a implantação destes programas, a infecção por via transfusional ainda representa um risco de contaminação de aproximadamente 15 a 25\%, sendo a identificação e a exclusão dos soropositivos assintomáticos e crônicos o grande desafio dos hemocentros. Depois da transmissão vetorial, a transfusão sanguínea é a segunda forma mais significante de transmissão da doença de Chagas na América Latina. No entanto, diante da erradicação do vetor e das melhorias nos serviços hemoterápicos, acredita-se que, na próxima década, a infecção chagásica entre doadores apresentará percentuais próximos de zero (BLEJER; SANGUIER; SALAMONE, 2001; BECERRIL et al, 2005; MORAES-SOUZA et al., 2006).

Para diagnóstico de doença de Chagas os hemocentros normalmente realizam a triagem clínica e a triagem sorológica. No Brasil os bancos de sangue utilizam como teste de escolha, preconizado pela ANVISA RDC 57/2010, o teste ELISA para realizar a triagem sorológica da doença. Este teste apresenta-se sensível, específico, de leitura objetiva e fundamenta-se na detecção de anticorpos anti-T. cruzi (SILVA; LUNA, 2013; BRASIL 2013).

Levando-se em consideração que a OPAS divulga que índices de prevalência de doadores inaptos para Chagas em torno de 0,20\% são aceitáveis para o Brasil (OPAS 2006), podemos analisar os índices das prevalências descritos na Tabela 1. Dessa forma, as maiores prevalências de doadores inaptos com sorologia positiva para doença de Chagas ocorreram na região Nordeste, especificamente na cidade de Iguatu-CE e na região Sudeste, na cidade de Patos de Minas-MG, com percentuais de 1,90\% e 1,20\% respectivamente. A alta prevalência especificamente na cidade de Iguatu-CE (1,90\%), certamente deve-se ao fato de aproximadamente $63,9 \%$ dos doadores regulares do Hemocentro local já terem doado sangue sem a realização de testes sorológicos. Essa prática foi realizada na região antes do 
funcionamento do Hemocentro (3 anos antes da pesquisa), confirmando a existência de doadores soropositivos para doença de Chagas na região sem os mesmos saberem de sua real condição. Outro fator a ser considerado está relacionado ao desconhecimento do potencial patogênico do inseto vetor pelos moradores soropositivos da região investigada, cerca de $73,8 \%$ afirmam que conheciam o inseto, denominado "procotó", mas não sabiam que o mesmo transmitia a doença. Este estudo também relata que a região passou por problemas no programa de combate ao vetor, com a falta de inseticida e pessoal para realizá-lo devidamente (SOBREIRA et al., 2001). Diante destes dados pode-se presumir que se não houve triagem sorológica adequada no serviço de hemoterapia e o combate ao vetor apresentou falhas nestas regiões, a inaptidão de doadores é certamente esperada.

Ao compararmos as 3 regiões descritas na Tabela 1 é possível visualizar que o maior número de publicações científicas avaliando a inaptidão sorológica para a doença de Chagas em hemocentros foram realizadas na região Sudeste. Sendo assim, podemos verificar que a maioria das pesquisas apresenta prevalências abaixo do índice de prevalência de doadores chagásicos previstos para o Brasil que é de 0,20\%. A soropositividade abaixo do índice encontrada na maioria dos municípios pode ser justificada pelo fato das cidades pesquisadas não serem regiões endêmicas para doença de Chagas, bem como pelo combate sistemático ao vetor nestas regiões (MORAES-SOUZA et al., 2006; OPAS 2006; FERREIRA-FILHO et al., 2011). À medida que se consegue manter a interrupção da transmissão por via vetorial, presume-se que ocorra uma diminuição gradativa da transmissão transfusional, que tende a ser mais controlada visando melhorar a qualidade do sangue. Uma vez que a incidência da doença de Chagas for reduzida, cada vez será menos frequente a possibilidade de se incluir chagásicos no grupo etário de doadores. Outro motivo que provavelmente explicaria a baixa prevalência de inaptidão por Chagas seria pela detalhada triagem clínica realizada junto aos possíveis doadores nos bancos de sangue destes locais (LUNARDELLI et al., 2007; FERREIRA-FILHO et al., 2011).

Conforme a Tabela 1, a região Sul do país apresentou percentuais de prevalência para doadores inaptos positivos para doença de Chagas variando entre 0,33\%, 0,40\% e 0,47\%, percentuais estes que estão próximos aos índices descritos nos hemocentros no Brasil $(0,20 \%)$. Essas prevalências são reflexo, sobretudo, do sucesso de políticas de saúde nacionais, estaduais e regionais indicando competência da região no controle da doença, assim como uma eficiente triagem clínica e sorológica nos serviços de hemoterapia locais (SOBREIRA et al., 2001; LUNARDELLI et al., 2007). 
Tabela 1. Prevalência de doadores inaptos com sorologia positiva para doença de Chagas em serviços de hemoterapia no Brasil por regiões, no período de 2000 a 2013.

Inaptidão relacionada a Doença de Chagas

\begin{tabular}{|c|c|c|c|c|c|}
\hline Regiões & Cidades/Estados & Doações de sangue & $\mathbf{N}^{\mathbf{0}}$ & $\%$ & Autores \\
\hline \multirow[t]{3}{*}{ Nordeste } & Pernambuco - PE & 743.529 & 499 & $0,07 \%$ & MELO et al., 2009 \\
\hline & Iguatu - CE & 3.232 & 61 & $1,90 \%$ & SOBREIRA et al., 2001 \\
\hline & Recife - PE & 204.124 & 31 & $0,01 \%$ & SABINO et al., 2010 \\
\hline \multirow[t]{9}{*}{ Sudeste } & Araraquara - SP & 49.541 & 19 & $0,04 \%$ & FERREIRA-FILHO et al., 2011 \\
\hline & Uberaba - MG & 79.729 & 116 & $0,15 \%$ & MORAES-SOUZA et al., 2006 \\
\hline & Patos de Minas - MG & 21.787 & 259 & $1,20 \%$ & SILVA; SILVA, 2010 \\
\hline & Botucatu - SP & 108.747 & 371 & $0,34 \%$ & NAVARRO et al., 2013 \\
\hline & Minas Gerais - MG & 262.300 & 196 & $0,07 \%$ & OLIVEIRA et al., 2009 \\
\hline & Uberaba - MG & 295.850 & 541 & $0,20 \%$ & LIMA et al., 2012 \\
\hline & Uberaba - MG & 95.990 & 129 & $0,13 \%$ & FERREIRA-SILVA et al., 2010 \\
\hline & Belo Horizonte - MG & 139.429 & 46 & $0.03 \%$ & SABINO et al., 2010 \\
\hline & São Paulo - SP & 271.880 & 141 & $0,05 \%$ & SABINO et al., 2010 \\
\hline \multirow[t]{3}{*}{ Sul } & Porto Alegre - RS & 36.720 & 122 & $0,33 \%$ & FITARELLI; HORN, 2009 \\
\hline & Porto Alegre - RS & 8.228 & 31 & $0,40 \%$ & LUNARDELLI et al., 2007 \\
\hline & Pelotas - RS & 4.482 & 21 & $0,47 \%$ & ARAÚJO et al., 2008 \\
\hline
\end{tabular}


Quanto às demais regiões do país, Norte e Centro-Oeste, não foram encontrados artigos na literatura científica nacional ou internacional pautados na temática abordada dentro do critério de inclusão utilizado para este estudo. Dessa forma, torna-se importante sugerir que estudos sobre a prevalência de doadores inaptos com sorologia positiva para doença de Chagas possam ser desenvolvidos nestas regiões colaborando com a complementação dos dados disponíveis no país relacionado a este evento no que tange à saúde pública.

A Figura 1 mostra a menor e a maior prevalência de sorologia positiva para T.cruzi em candidatos à doação de sangue verificada nas três regiões pesquisadas. A menor e a maior prevalência foram verificadas em Recife-PE e Iguatu-CE com um percentual de 0,01\% e $1,90 \%$ respectivamente, ambas na região Nordeste. Ao avaliarmos essa discrepância de prevalências na mesma região podemos inferir que se trata de aspectos particulares de triagem clínica e sorológica no hemocentro e combate ao vetor de cada localidade, não sendo possível avaliar a região de modo amplo. Acredita-se que a baixa prevalência verificada em Recife-PE $(0,01 \%)$ possa ser explicada entre outros aspectos, pela combinação do sucesso do controle do vetor com o aumento da urbanização da população no Brasil, uma vez que doadores jovens não viveram em zona rural nem foram expostos ao vetor (SABINO et al., 2010). E, conforme discutido, a falha da triagem sorológica bem como o inadequado combate ao vetor, elevou a prevalência no município de Iguatu-CE (SOBREIRA et al., 2001).

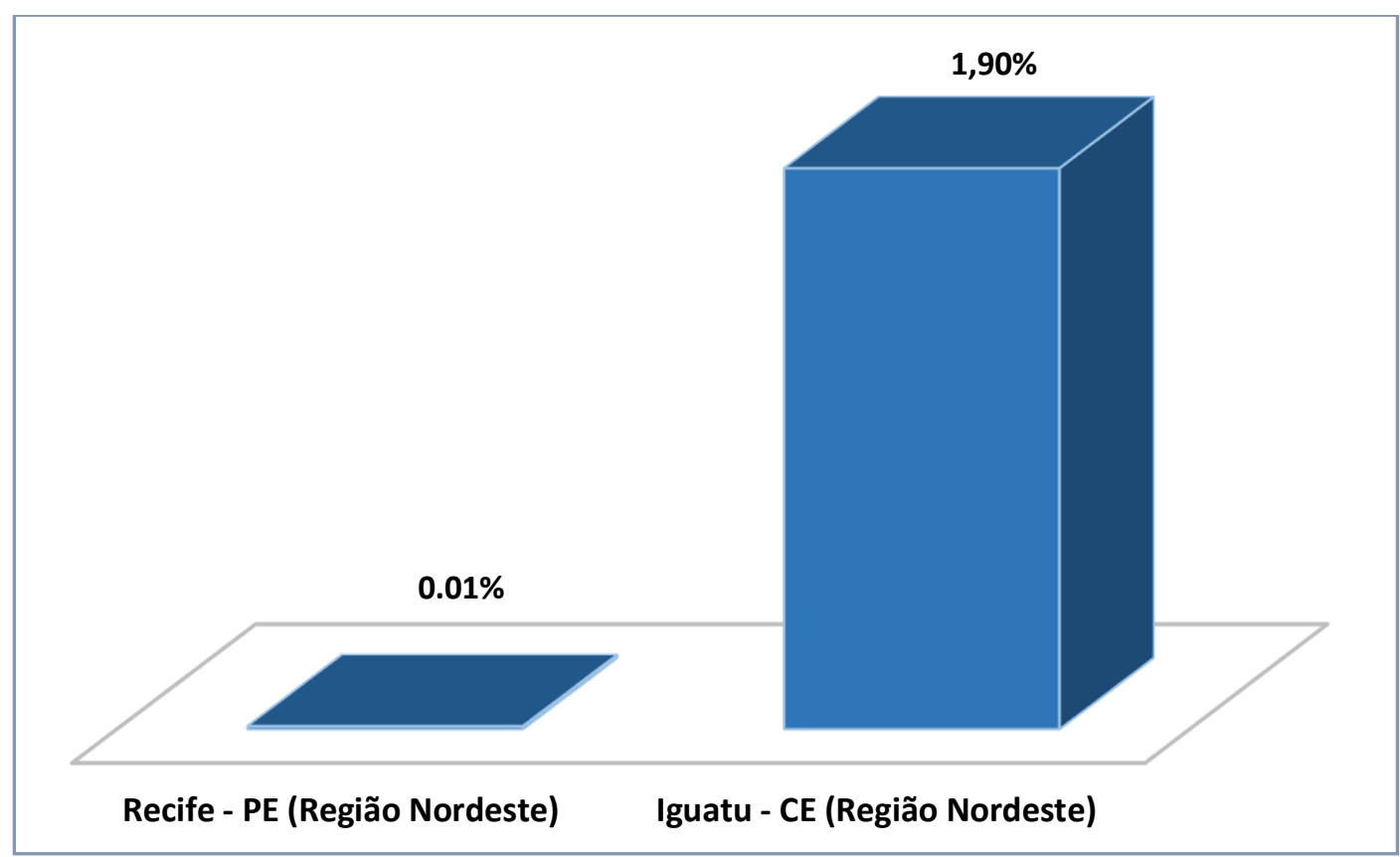

Figura 1. Menor e maior prevalência de sorologia positiva para T.cruzi em candidatos à doação de sangue verificada nas 3 regiões pesquisadas. 


\section{CONCLUSÃO}

A realização de pesquisas comparando a prevalência de doadores inaptos com sorologia positiva para doença de Chagas em bancos de sangue nas diferentes regiões do Brasil é de extrema relevância para avaliar a "situação transfusional" peculiar dos hemocentros de cada região. A determinação das prevalências pode ser relevante como indicadores do risco da doença de Chagas transfusional bem como para julgar o nível de transmissão da doença em uma determinada região. Dessa forma, é possível traçar estratégias locais que envolvam os esforços de todos os setores relacionados na área como vigilância sanitária, hemocentros e laboratórios, unidos para eliminar a transmissão transfusional da doença de Chagas e melhorar a qualidade do sangue transfundido.

\section{REFERÊNCIAS BIBLIOGRÁFICAS}

AGÊNCIA NACIONAL DE VIGILÂNCIA SANITÁRIA. Ministério da Saúde. Resolução da Diretoria Colegiada n. 57, 16 de dezembro de 2010. Disponível em: <http://www.prosangue.sp.gov.br/uploads/legislacao>. Acesso em: 27 de ago. 2014.

ARAS, R.; GOMES, I.; VEIGA, M.; MELO, A. Transmissão vetorial da doença de Chagas em Mulungu do Morro, Nordeste do Brasil. Rev. da Sociedade Brasileira de Medicina Tropical. v. 36, n. 3, p. 359-363. 2003.

ARAÚJO, A. B.; VIANNA, E. E. S.; BERNE, M. E. A. Anti-Trypanosoma cruzi Antibody Detection in Blood Donors in the Southern Brazil. The Brazilian Journal of Infectious Diseases. v. 12, n. 6, p. 480-482. 2008.

BARBOSA, L. G. N. Doença de Chagas. Revista Logos. n. 1, p. 20-36. 2009.

BECERRIL, NH; MEJÍA, AM; VERDUGO, MAB; MURILLO, VG; TOQUERO, EM; LÓPEZ, R; TREVETHAN, S; CARDENAS, M; REYES, PA; HIRAYAMA, K; MONTEÓN, VM. Blood transfusion and iatrogenic risks in Mexico city. Anti-Trypanosoma cruzi seroprevalence in 43048 blood donors, evaluation of parasitemia, and electrocardiogram findings in seropositive. Mem Inst. Oswaldo Cruz, v. 100, n. 2, p. 111-116. 2005.

BLEJER, J.L.; SAGUIER, M.C; SALAMONE, H.J.; Antibodies to Trypanosoma cruzi among blood donors in Buenos Aires, Argentina. Int. J. Infect Dis., v.5, p. 89-93. 2001. 
BRASIL, Ministério da saúde. Notícias. 2006. Disponível em < http://www. saude. gov.br. >. Acesso em 15 set. de 2014.

BRASIL, Ministério da Saúde. Portaria n. ${ }^{\circ}$ 2.712, de 12 de Novembro de 2013. Redefine o regulamento técnico de procedimentos hemoterápicos. Diário Oficial da União, 13 nov. 2013, n. 221, Seção 1, p. 106. Disponível em < http://bvsms.saude.gov.br/bvs/saudelegis/gm/2013/prt2712_12_11_2013.html >. Acesso em: 20 de out. de 2014.

CARRAZZONE, C. F. V.; BRITO, A. M.; GOMES, Y. M. Importância da avaliação sorológica pré-transfusional em receptores de sangue. Revista Brasileira de Hematologia e Hemoterapia, São José do Rio Preto, v. 26, n. 2, p. 93-98. 2004.

DIAS, J. C. P. Doença de Chagas e transfusão de sangue no Brasil: vigilância e desafios. Revista Brasileira Hematol. Hemoter., São José do Rio Preto, v.28, n. 2, p. 81-87, 2006.

FEREIRRA FILHO, J. C. R.; COSTA, P. I.; BUAINAIN, A.; ROSA, J. A. Soropositividade para doença de Chagas entre doadores de sangue em Araraquara, Estado de São Paulo, no período de 2004 a 2008. Revista da Sociedade Brasileira de Medicina Tropical, Uberaba, v. 44, n. 1, p. 110-112, jan.-fev. 2011.

FERREIRA-SILVA, M. M.; PEREIRA, G. A.; LAGES-SILVA, E.; MORAES-SOUZA, H. Socioepidemiological screening of serologically ineligible blood donors due to Chagas disease for the definition of inconclusive cases. Mem. Inst. Osvaldo Cruz, v. 105, n. 6, p. 800-805, Rio de Janeiro. 2010.

FITARELLI, DOUGLAS B.; HORN, JOEL F. Descarte de bolsas de sangue devido à reatividade para doença de Chagas em um laboratório de triagem sorológica de doadores em Porto Alegre - RS. Revista Brasileira de Hematologia e Hemoterapia, São Paulo, v.31, n. 5, São Paulo, 2009.

LIMA, L. M.; ALVES, N. P.; BARBOSA, V. F.; PIMENTA, G. A.; MORAES-SOUZA, H.; MARTINS, P. R. J. Prevalence of Chagas disease in blood donors at the Uberaba Regional Blood Center, Brazil, from 1995 to 2009. Revista da Sociedade Brasileira de Medicina Tropical. v. 45, n. 6, p. 723-726, nov. -dez. 2012.

LOZANO, V. F. Avaliação da atividade antiparasitária e efeito sinérgico de compostos cumarínicos comparados ao benzonidazol em duas cepas de Trypanosoma cruzi. São Paulo. Dissertação. Universidade Bandeirantes de São Paulo. 2011. 
LUNARDELLI, A.; BORGES, F. P.; MELLO, K. F.; ZEFERINO, A. S. A. Soroprevalência da doença de Chagas em candidatos a doadores de sangue. Revista Brasileira de Análise Clínica, v. 39, n. 2, p. 139-141. 2007.

MELO, A. S.; LORENA, V. M. B.; MORAES, A. B.; PINTO, M. B. A.; LEÃO, S. C.; SOARES, A. K. A.; GADELHA, M. F. S.; GOMES, Y. M. Prevalência de infecção chagásica em doadores de sangue no estado de Pernambuco, Brasil. Revista Brasileira de Hematologia e Hemoterapia, São Paulo, v.31, n. 02, p. 69-73. 2009.

MEYER, I. F.; KANESHIMA, E. N.; SOUZA-KANESHIMA, A. M. Alterações no sistema digestivo desencadeadas pelo quadro infeccioso do trypanosoma cruzi. Iniciação Científica Cesumar, v. 08, n. 01, p. 11-23, Jan-jun. 2006.

MORAES-SOUZA, H.; MARTINS, PAULO R. J.; PEREIRA, GILBERTO A.; FERREIRASILVA, MÁRCIA M.; ABUD, MURILO B. Perfil sorológico para doença de Chagas dos doadores de sangue do Hemocentro Regional de Uberaba. Revista Brasileira de Hematologia e Hemoterápico, São José do Rio Preto, v. 28, n. 2, p. 110-114. Abr.-jun. 2006.

MORAES-SOUZA, H.; FERREIRA-SILVA, M. M. O controle da transmissão transfusional. Rev. História sobre a Doença de Chagas no Brasil, Uberaba, v. 44, supl. II, p. 64-67. 2011.

NAVARRO, E. C.; GOTO, R. L.; RICOBONI, I. S.; CORENTE, J. E.; HENRIQUES, R. M. S.; NEVES, S. L.; ZANINI, J. M.; DORINI, A. A.; PEREIRA, P. C. M. Soroprevalence of chagasic infection in Young individuals in a blood center in the state of São Paulo, Brazil. Revista Inst. Med. Trop., São Paulo, v. 55, n. 4, p. 245-250, jul-agu. 2013.

OLIVEIRA, C. D. L.; LOUREIRO, F.; BASTOS, M. R. D.; PROIETTI, F. A.; CARNEIROPROIETTI, A. B. F. Blood donor deferral in Minas Gerais State, Brazil: blood centers as sentinels of urban population health. Transfusion, v. 49, n. 5, p. 851-857. 2009.

ORGANIZACIÓN PANAMERICANA DE LA SALUD. Estimación cuantitativa de la enfermedad de Chagas en las Américas (OPS/HDM/CD/425-06). 2006.

RAMOS, JR. A. N.; CARVALHO, D. M. Os diferentes significados da certificação conferida ao Brasil como estando livre da doença de Chagas. Caderno de Saúde Pública. Rio de Janeiro, v.17, n. 6, p. 1403-1412. 2001.

SABINO, E.C.; SALLES, N.A.; SARR, M.; BARRETO, A.M.; OIKAWA, M.; OLIVEIRA, C.D.; LEAO, S.C.; CARNEIRO-PROIETTI, A.B.; CUSTER, B.; BUSCH, M.P. Enhanced classification of Chagas serologic results and epidemiologic characteristics of seropositive donors at three large blood centers in Brazil. Transfusion, v. 50, n. 12, p. 2628-2637, 2010. 
SANTOS, C. A. T.; Infecção pelo Trypanosoma cruzi em doadores de sangue: revisão sistemática da literatura e estudo de prevalência no hemocentro de Goiás. Goiânia-Go. Dissertação de Mestrado em Ciência da Saúde. Universidade Federal de Goiás. 2011.

SAÚDE-GUIMARÃES, D. A.; FARIA, A. R. Substâncias da natureza com atividade antiTryppanosoma cruzi. Revista Brasileira de Farmacognosia, João Pessoa, v. 17, n. 3, p. 455465, jul.- set. 2007.

SILVA, P. L.; SILVA, R. M. G. Inquérito soroepidemiológico sobre a infecção chagásica em doadores de sangue na região do Alto Paranaíba, Minas Gerais. Bioscience Journal, Uberlândia, v. 26, n. 5, p. 824-827, set. - out. 2010.

SILVA, V. L. C.; LUNA, E. J. A.. Prevalência de infecção pelo T. cruzi em doadores de sangue nos hemocentros coordenadores do Brasil em 2007. Revista Epidemiologia e Serviços de Saúde, Brasília v. 22, n. 01, p.103-110. Mar. 2013.

SOBREIRA, A. C. M.; GOMES, F. V. B. A. F.; SIlVA, M. A. M.; OlIVEIRA, M. F. Prevalência de infecção chagásica em doadores de sangue do Hemocentro Regional de Iguatu, CE. Revista da Sociedade Brasileira de Medicina Tropical, Uberaba, v. 34, n. 2, p. 193 196, mar-abr. 2001.

WHO. Chagas disease (American trypanosomiasis). Geneve, 2010. Disponível em < http://www.who.int/mediacentre/factsheets/fs340/en/ >. Acesso em: 17 de out. de 2014. 